\title{
Comparison Between Ipv4 And Ipv6 Using Opnet Simulator
}

\author{
Ghaida Yagoub Ahmed Yosif Al-Gadi ${ }^{1}$, Dr. Amin Babiker A/Nabi Mustafa ${ }^{2}$ \\ Ahmed Yagoub Ahmed Yosif Al-Gadi ${ }^{3}$ \\ 1,2 \\ Faculty Of Engineering, Neelain University ,Khartoum -Sudan \\ ${ }^{3}$ Faculty Of Engineering Sudan University ,Khartoum-Sudan
}

\begin{abstract}
The overall objective of the paper is to evaluate and compare the performance of IP (IPv4 and IPv6), using OPNET [1] as a simulation tool to compare them in terms of different criteria. In this, we have designed a wide area networks based on IPv4and the IPv6 protocols in OPNET, a network simulator platform. Simulation techniques allow Lena analyze the behavior of each network separately. The network consists of different components such as servers, routers, and adapters. The drive of this paper is to assess the basic productivity, and packet loss, a delay, jitter, and the use of methods based on simulative and analytical network addresses in IPv4 / IPv6. The aim of this paper is to find out what is the best IP under a particular application. Understanding the impact of the delay, and the wrath of use and productivity in the network addresses of IPv4/IPv6.. The simulation has been carried out using Optimized Network Engineering Tool (OPNET).
\end{abstract}

Keywords: - IPv4, IPv6, OPNET, delay, throughput, jitter.

\section{INTRODUCTION}

The great expansion of the internet, these days, creates more significant challenges. Not only the addressing of new hosts like computer, tablets, laptop, cell phone but also the technologies. Requires that its overall architecture evolve to accommodate the new technologies that support the growing demand for use (by users, application, or services).

Internet Protocol Version 6 (IPv6) [2], is designed to meet these requirements and enable a global environment where the addressing rules of the network are again transparent to the applications. IPv6 makes important improvements to network topologies dynamically (exp. Peer to peer, client/server or mesh networks). Also, it improves most of networks functions, notably in security, mobility, auto configuration, quality of service (QOS) and multicasting [3].

IPv6 is proposed to provide Internet with a larger address space and better performance [4].

As a result, Significant progress in the communications revolution asks us to commit all devices to be in constant contact, continuous and high quality and taking into account the piece. In this paper, we have two types of networks designed for IPv4 and IPv6. In an attempt to understand the comparison between both of the Protocols in a simulated environment OPNET [1].

\section{NETWORK ARCHITECTURE OF IPV4 AND IPV6 IN OPNET}

Using the OPNET simulation tool the editor designed an IPv4 and IPv6. Taken a set of parameters that describe the features of the work, and the differences between the performances of each of the protocols. . All attached to the results in order to highlight the fundamental differences between network protocols both IPv4 and IPv6, has been designing networks and analyze different aspects in terms of the parameters (delay, throughput, response time and jitter), have emerged showing the attributes of these protocols.

\section{IPv4/IPv6Design and Implementation:}

We have used OPNET software tosimulates IPv4 and IPv6, and to measure the performance of the network using eachprotocols according to four parameters( dela, ,jitte, utilization and throughput. . The objective of this simulation to compare between IPv4 and IPv6 by using two scenarios of WAN network Containing two local networks (LAN each containing 14 Ethernet _weakestn and 1 Ethernet_server. . Each local network, is linked by a router, and the two routers are linkedd 2 by ppp-sd 3 show figure. $(1,2)$. 


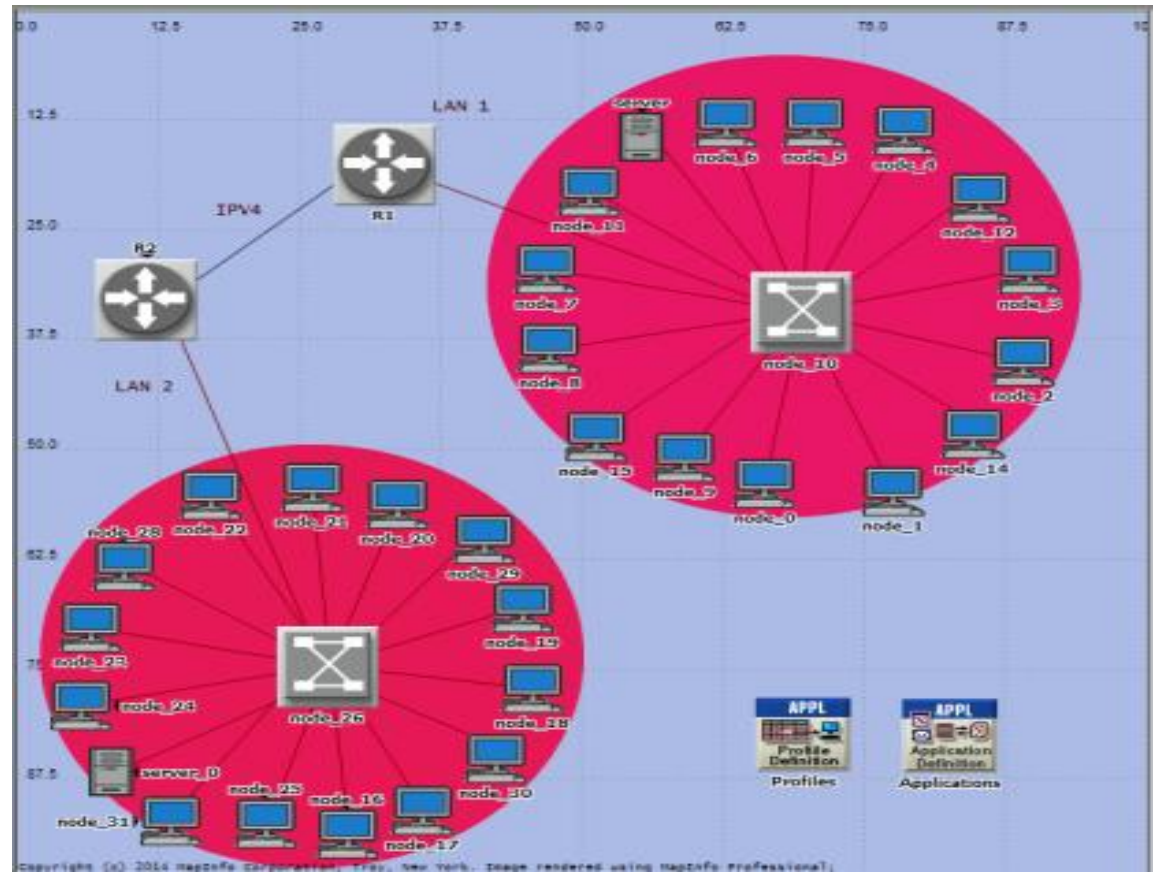

Figure 1. IPv4 Architecture

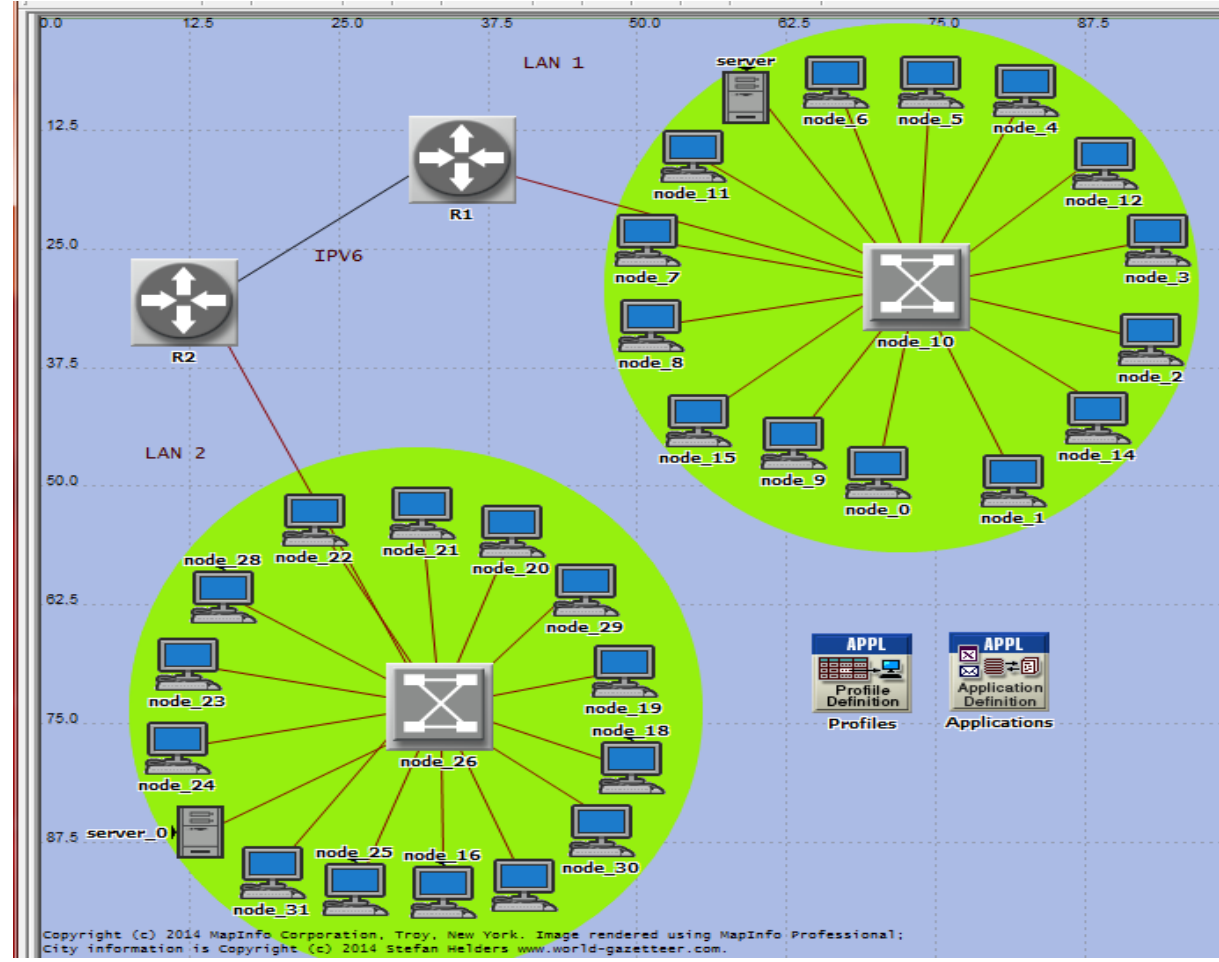

Figure 1. IPv6 Architectur

\section{METHOD}

The method consisted of a selection of one server, 14 Ethernet workstations Ethernet switches, and a router. This selection represents one office. In this case, the 14 workstations and 1 server are connected to the switch via Ethernet 100baseT. Each office has one router connected to the switch. The two offices are connected to each other, using the ppp - ds3 link connecting the two routers. After establishing the connection using the above mentioned method, we defined the application using Application definition to set which application do we need to apply in this scenario. In this case we need to apply voice, http, and Email in our scenario. 
After that we defined the profile using Profile definitions to set which profile do you need to apply. In this case we need to apply voice application, http and Ethernet. To do that, in free space right click and choose individual DES statistics, then pick which parameter does your analysis in your scenario, in this case we select Ethernet delay and node delay and throughput, jitter, response time (http), voice (traffic send and receive) and, utilization. IPv6 addresses in the network by using the Protocols >IPv6> Auto-Assign IPv6 Addresses option and IPv4 addresses in the network by using the Protocols >IPv4>Auto-Assign IPv4 Addresses [5].

On free space right click and choose individual DES statistics and choose which parameter do your analysis in your scenario, in this case we select Ethernet delay and node delay.

\section{A. Network components:-}

This section discusses the following network components used in the suggested network models running on OPNET [6].

- The "Profile Config" node: -Can be used to create profiles for users, which can be applied to different nodes in the network, that is used to generate layer 7 (application layer) traffic. In the "ApplicationConfig" you must crate application to be used by this profile.

- Using the "Application Config" object before using this object. You can specify the traffic patterns followed by the applications as well as the configured profiles of this object.

- The Ethernet _ wkstn node model: -represents a workstation with client-server applications running over TCP, and UDP. The workstations support only one Ethernet connection at $10 \mathrm{Mbps}, 100 \mathrm{Mbps}$, or $1000 \mathrm{Mbps}$. This workstation requires a fixed amount of time to route each packet, as determined by the "IP Forwarding Rate" feature of this node. Packets are routed on FCFS (First come, first serve) basis. It might also encounter queues at the lower layers of the protocol inasmuch it depends on transmission rates of corresponding interface output.

- Router type ethernet4_slip8_gtwynode object

- The Ethernet_server model represents a server node with server applications running over TCP and UDP.

- The 100BaseT link: -Links between hosts and routers or hosts and switch. Represents an Ethernet connection operating at $100 \mathrm{Mbps}$. It can connect any combination of the following nodes (except switch to router, which connect hub to hub or switch to switch or switch to work station.

- PPP-DS3 duplex link: -Links between routers. Packet Formats:ip3_dgram.Data Rate:DS3 (44.736 Mbps).

Supports one underlying Ethernet connection at $10 \mathrm{Mbps}, 100 \mathrm{Mbps}$, or $1 \mathrm{Gbps}$. The operational speed is determined by the connected link's data rate.

\section{RESULT}

Figure 3 below shows the comparison of Ethernet delay between IPv4 and IPv6and shows also both types of theoretical have transient state and stead state station and the IPv6 has small stead stated time if we compare it with IPv4.

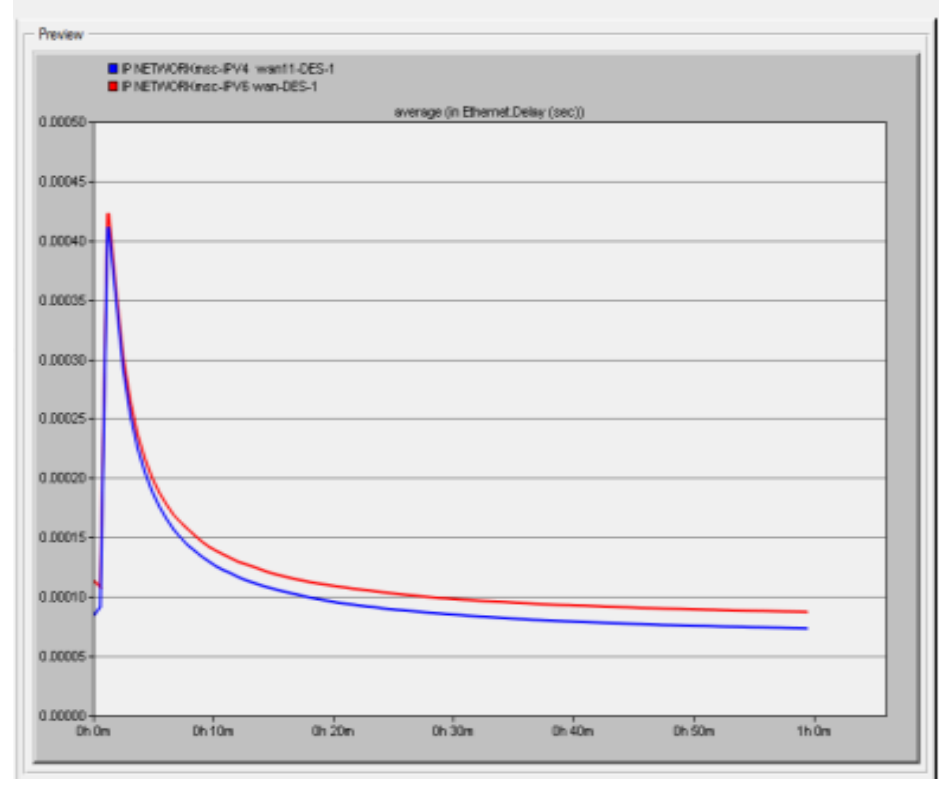

Figure 3. Ethernet Delay 
Fragmentation in IPv6performed by the host only. For this reason the delay of IPv6 is bigger than the delay in IPv4but with a small difference. The IPv6 has a higher Delay than IPv4because IPv6hasa larger header field. IPv6has a smaller header field, the curve takes latency of the time in the beginning before the Guide to up, because the devices in the state start on then curve Guide to high because the devices are requesting at the same time. Delay has inverse relationship the number of devices.

Figure 4 shows the Email Download Response Time.

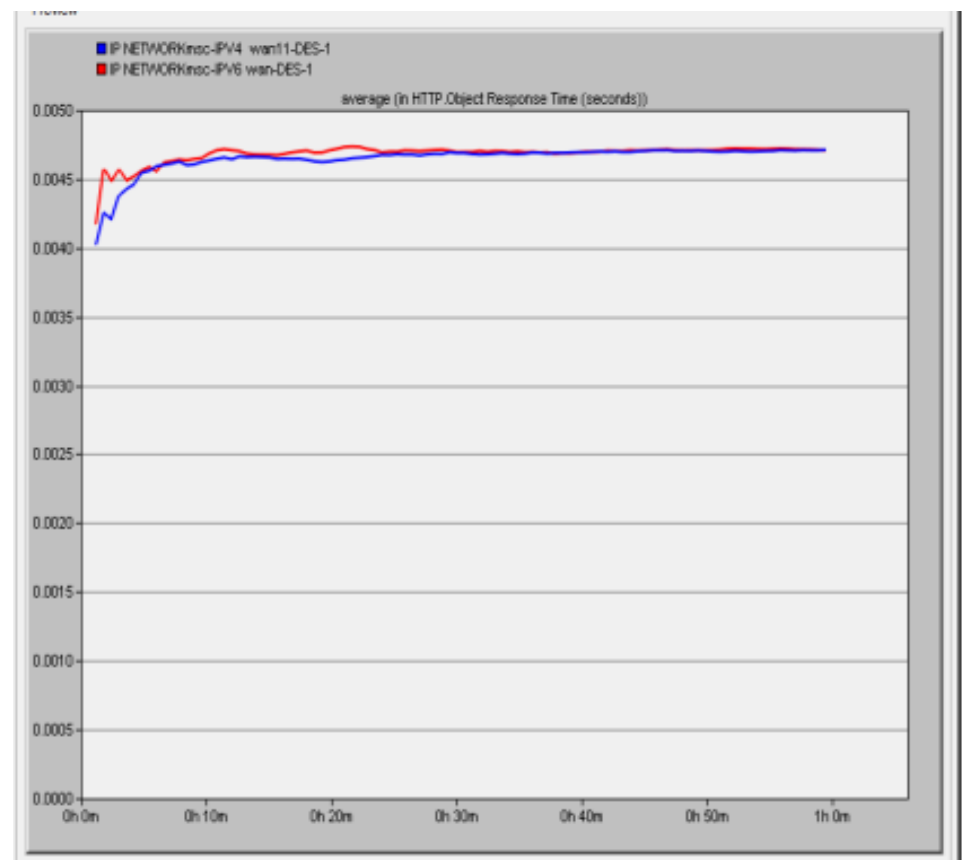

Figure 4. Http Download Response Time

The Response time is measured from the time a client application sends a request to the server until it receives the response packet. Data traffic gradually increases the response time forIPv4 network. The difference in Http download response time of anIPv4and an IPv6 network is small.

Figure 5 below show the comparison of voice jitter.

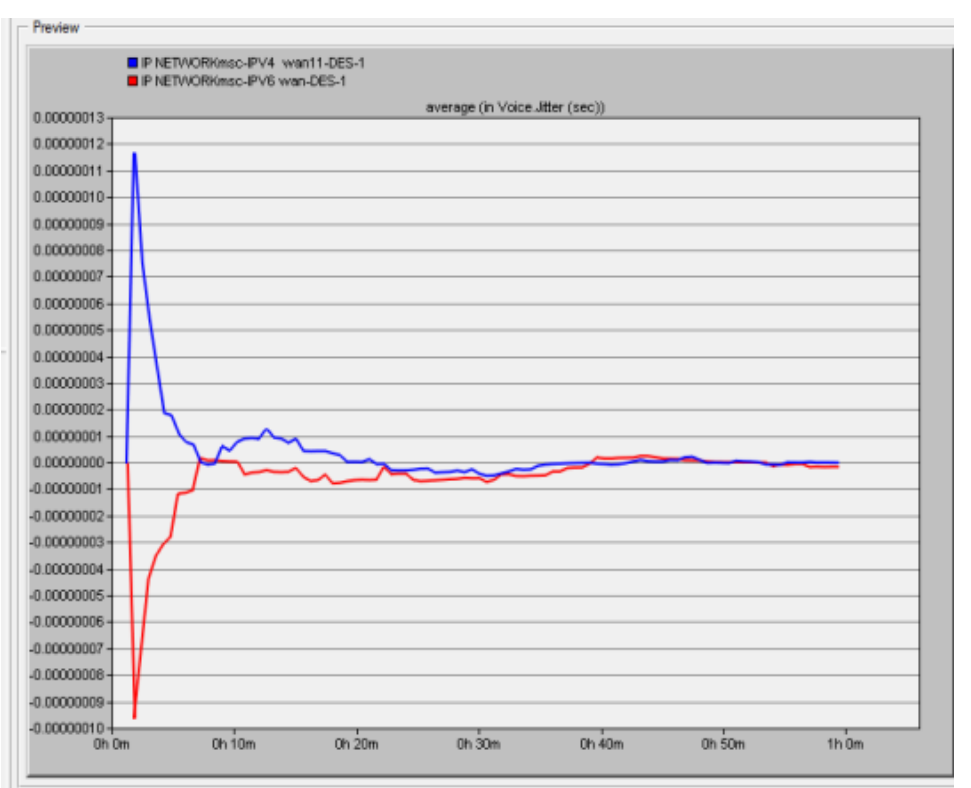

Figure 5: Voice Jitter

Jitter non uniform delays, requires buffering at the endpoints and application level reordering. Because of the increased jitter, it is not easy to tell if the packet is missing, lost or just late. IPv4have higher jitter than IPv6. 
Figure 6,7 below shows the comparison of IPV4 and IPV6voicetrafficc sendand receiveebetweenn IPv4 and IPv6.

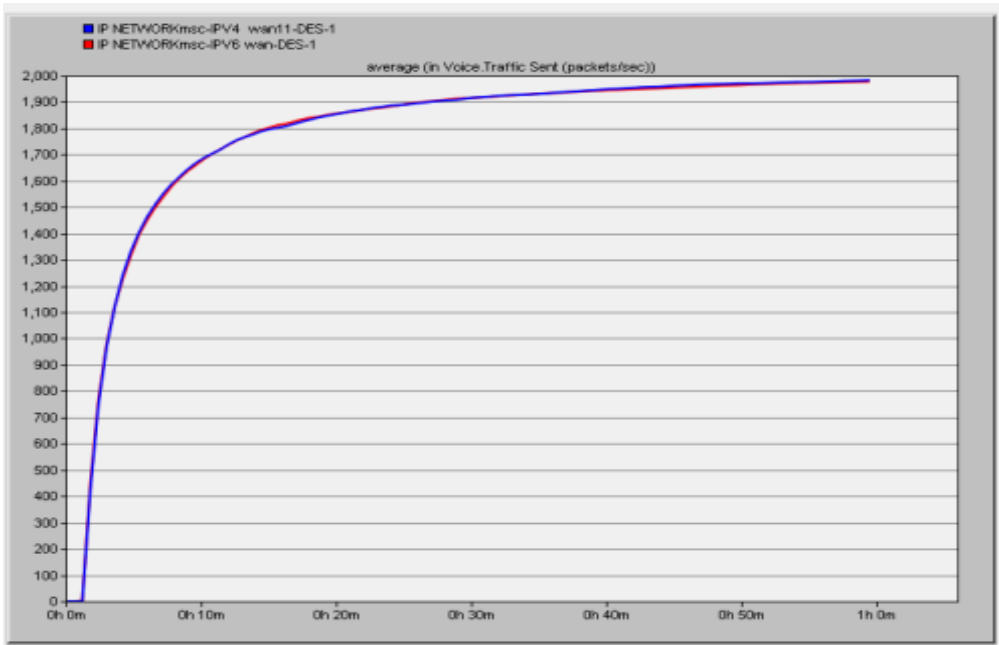

Figure 6. IPV4 and IPV6 voice traffic send

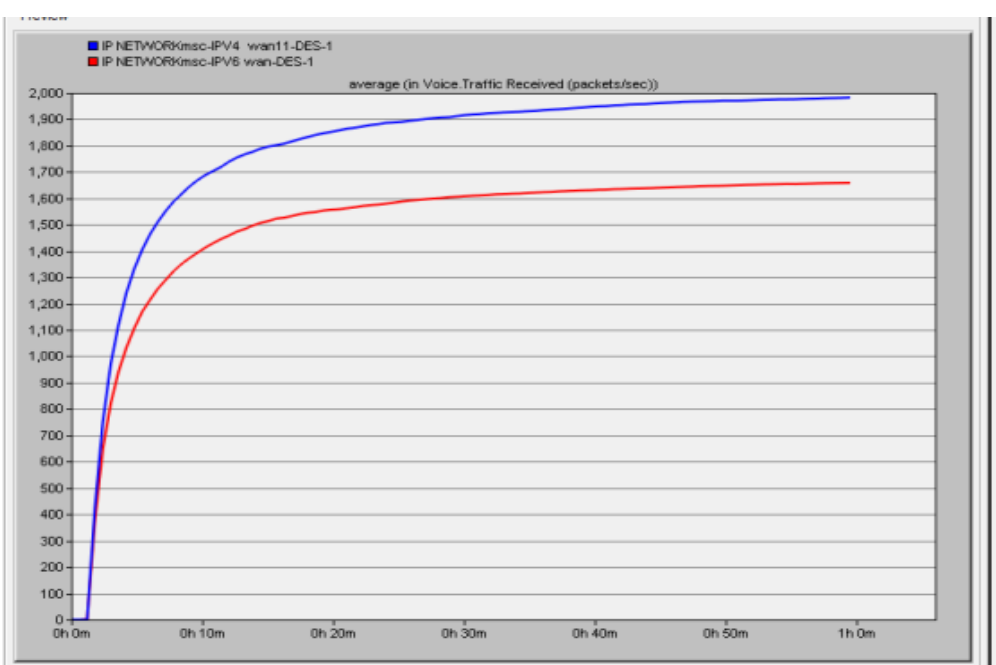

Figure 7. IPV4 and IPV6 voice traffic receive

In figure 8there are few differences between IPv4 network and IPv6 network in Email download response second.

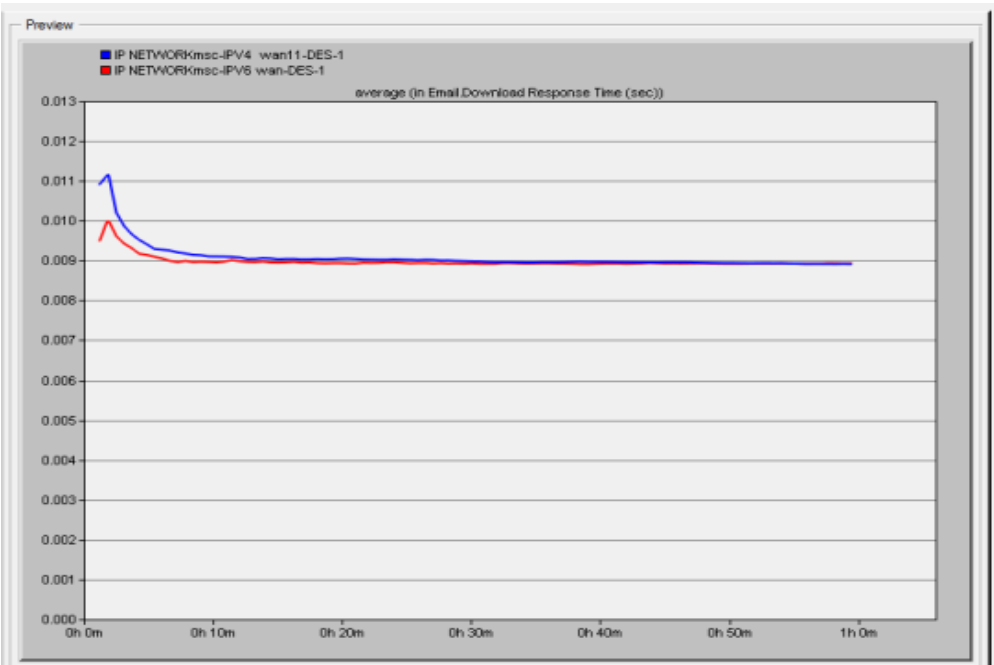

Figure 8. Email Download Response Time 
Figure 8 shows the Email Download Response Time The broad objective is to evaluate and compare the performance of two protocol stacks (IPv4 and IPv6) parameters that have to be analyzed when the data is being transmitted from one client to another or to a server over a wired network. In this case we have designed wired networks on the basis of IPv4 and IPv6 protocols.

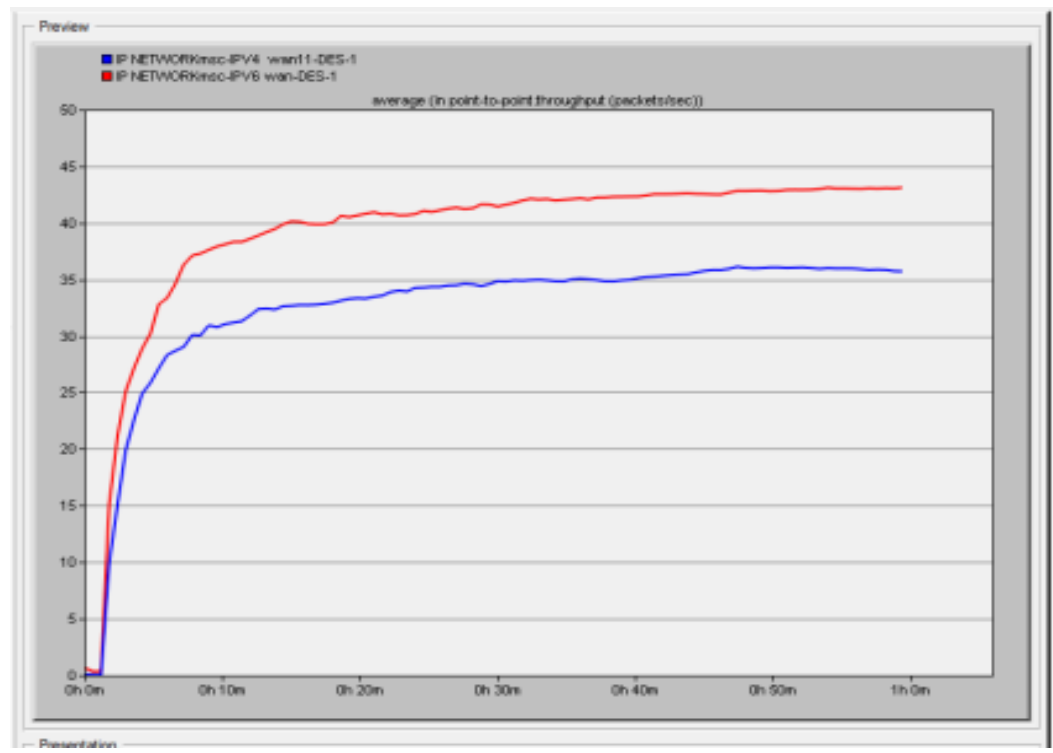

Figure 9.point to point Utilization

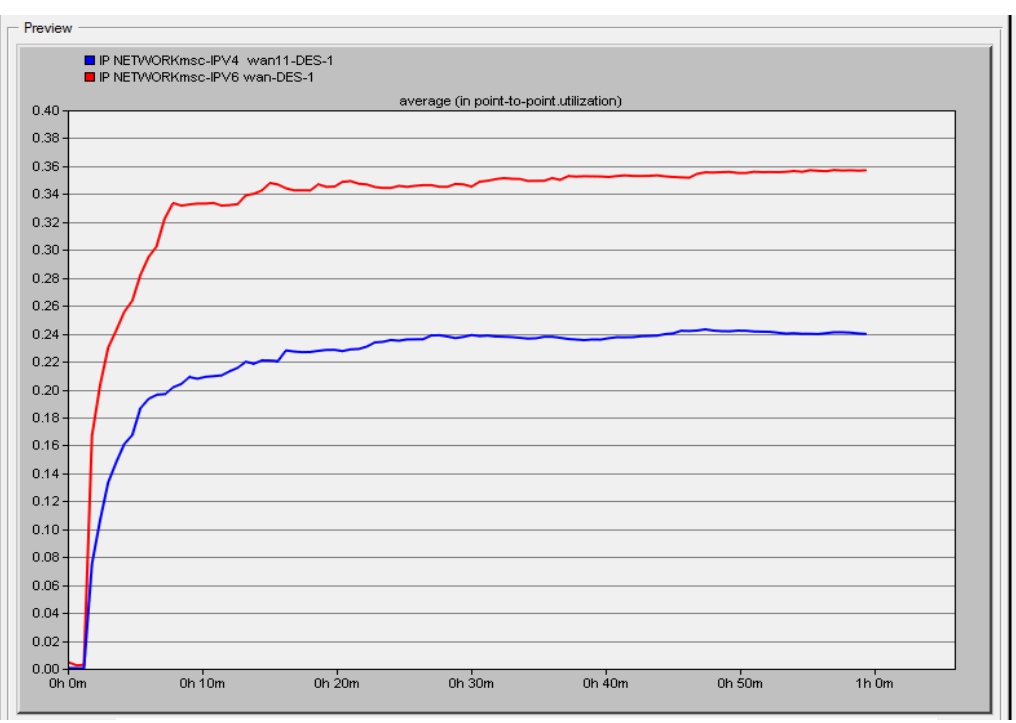

Figure 10.point to point throughput

Figure 10 below is representative of freemen figure 9 the sizes with a throughput value of $100 \%$ for all IPv4/IPv6 traffic ratios. The CPU steadily increases since the rate line rises. The top most utilization per line rate are those traffic mixes with the most IPv6 traffic.

CPU utilization increases when a system process requires more time or when more network packets are sent and received. Under normal operating conditions, on a no stackable switch, the CPU is busy at least 5 percent of the time. If the switch is stacked, the CPU is busy at a minimum of ( 7 or 8$) \%$ utilization. The CPU utilization in a switch stack is measured on the master switch only. However, this is directly affected by the number of stack members.

Figure 10 below shows the comparison of point to point throughput between IPv4 and IPv6. 


\section{CONCLUSION}

In this thesis paper, we have evaluated the Ethernet delay of low load (voice) and medium load (http) and heavy load on IPv4/IPv6 networks. Two network scenarios calledIPv4 network and IPv6 network have been simulated. The simulation has been carried out by using OPNET. Comparative investigation of Ethernet delay based on simulative and analytical approaches was carried in both network scenarios. Based on this research, research question was aimed to understand and investigate what is the difference in the delay is experienced in IPv4 and IPv6 network or what is best for the user from $(\mathrm{QoS})$ site. The simulation result in low load note IPv4 is a lower delay than IPv6, in medium load the delay is very low between them in high load the delay is equal.

Thus, the analysis of IPv4 and IPv6 networks presents us with their performance characteristics through statistical analysis. The statistics obtained from simulation tells us that the performance of IPv6 is much better than IPv4. IPv6 performs better under specific circumstances. Insofar as the performance is concerned; the IPv6 protocol has better transmission efficiency and high throughput and the largest utilization per line rate are those traffic mixes with the most IPv6 traffic.

The IPv6 has a higher Delay than IPv4because IPv6hasa larger header field. IPv4 has a smaller header field and the packet frame. Another key aspect is the jitter. Jitter is basically a slight irregular directional flow of the electrical signals, which are actually the data packets. When the simulation was a running state, then more or less there was no major difference observed in the jitter values of both the protocols. Although in a comparison, IPv6 showed less jitter than IPv4 protocol. With the extinction of the address spaces in IPv4, there is an immediate need to adopt IPv6 protocol as early as possible, so as to avoid future impediments in the Internet network.

[1] "OPNET: IPv6 for R\&D Specialized Model" [Online] Available: http://www.opnet.com/solutions/network_rd/simulation_model_library/IPv6.html, Accessed on 15 December, 2013

[2] IP Version 6 Addressing Architecture, RFC 2373, R. Hinden, S. Deering, July 1998

[3] A.Dutta,J.ALberi,A.Cheng,B.Horgan,T.McAuley,D.Chee,B.Lyles,"IPv6TRANSITIONTECHNIQUSFO RLEGACY APPLICATION":582,MILCOM 2006.

[4] Ali, Amer nizar abu. Comparison study between IPv4\&IPv6. International journal of computer science issue. May 1, 2013.

[5] "OPNET Training" [Online] Available: https://www.opnet. Com/training/index.html, Accessed on 29 January 2013

[6] "OPNET: IPv6 for R\&D Specialized Model" [Online] Available: http://www.opnet.com/solutions/network_rd/simulation_model_library/IPv6.html, Accessed on 12 December, 2013. 